\title{
Single nucleotide variant sequencing errors in whole exome sequencing using the Ion Proton System
}

\author{
SHIRO FUJITA, KATSUHIRO MASAGO, CHIYUKI OKUDA, AKITO HATA, \\ REIKO KAJI, NOBUYUKI KATAKAMI and YUKIO HIRATA
}

Division of Integrated Oncology, Institute of Biomedical Research and Innovation, Chuo-ku, Kobe 650-0047, Japan

Received April 28, 2017; Accepted May 11, 2017

DOI: $10.3892 /$ br. 2017.911

\begin{abstract}
Errors in sequencing are a major obstacle in the interpretation of next-generation sequencing (NGS) results. In the present study, sequencing errors identified from analysis of single nucleotide variants (SNVs) identified during exome sequencing of human germline DNA were studied using the Thermo Fisher Ion Proton System. Two consanguineous cases were selected for sequencing using the AmpliSeq Exome capture kit, and SNVs found in both cases were validated using Sanger sequencing. A total of 98 SNVs detected by NGS were randomly selected for further analysis. Nine of the analyzed SNVs were shown to be false positives when confirmed by Sanger sequencing. All but one SNV were considered to be homopolymer regions, mainly through the insertion or deletion of nucleotides. The remaining error was considered to be related to the primer. The present results revealed that the majority of the SNV sequencing errors originated from homopolymer insertion/deletion errors, which are commonly observed when using the Ion Torrent system.
\end{abstract}

\section{Introduction}

Next-generation sequencing (NGS) has become a powerful and widely used clinical tool for the screening of mutations in hereditary disease and for the evaluation of driver and passenger mutations in cancer $(1,2)$. Whole exome sequencing (WES), which targets protein coding regions of the genome, is the preferred option for the finding of new causative genetic variants in rare Mendelian disorders

Correspondence to: Dr Shiro Fujita, Division of Integrated Oncology, Institute of Biomedical Research and Innovation, 2-2 Minatojima Minamimachi, Chuo-ku, Kobe 650-0047, Japan

E-mail: jp.shirofujita@gmail.com

Abbreviations: DNA, deoxyribonucleic acid; dNTP, deoxyribonucleotide triphosphate; ISPs, ion sphere particles; NGS, next-generation sequencing; PCR, polymerase chain reaction; SNV, single nucleotide variant; WES, whole exome sequencing

Key words: single nucleotide variants, next-generation sequencing, sequencing error, exome sequencing, Ion Torrent, Ion Proton as well as the identification of genetic variants associated with individual types of cancer $(3,4)$. The Ion Torrent NGS platform implements a semiconductor-based sequencing technology, the underlying principle of which is the non-optical detection of hydrogen ions released from the sequential addition of deoxynucleotides to a deoxyribonucleic acid (DNA) chain (5). The Ion Proton System, with $15 \mathrm{~Gb}$ output per run, and the AmpliSeq Exome kit, which performs target enrichment of the entire human exome by multiplex-polymerase chain reaction (PCR) amplification, enable researchers to examine exomes rapidly and with low cost.

However, sequencing errors remain one of the main obstacles in the identification of causative genetic variants and/or mutations. Several patterns of sequencing error are recognized based on the rationale of the NGS system. Compared to the Illumina platforms, the Ion Proton platform has a high ratio of false positives in the identification of small insertion and deletion mutations (indel) but shows high accuracy in the identification of single nucleotide variant (SNV) (6-8). Considering that the vast majority of mutations (>90\%) in human genome present as SNVs, and that clinical practice necessitates rapid sequencing at an acceptable cost for relatively few samples, the Ion Proton System is an attractive option for clinical exome evaluation. Therefore, information regarding SNV sequencing error (such as frequency and/or etiologic character) is important, as it has the potential to improve diagnostic accuracy and may avoid waste in the clinical decision process.

In the present study, we describe the incidence and characteristics of sequencing errors during WES with the Ion Proton System, confirmed by Sanger sequencing.

\section{Materials and methods}

Overview. Two consanguineous patients were selected for WES. Identified SNVs were validated with Sanger sequencing.

Ethics statement. The present study was approved by the Institute of Biomedical Research and Innovation Hospital's Institutional Review Board. All the patients provided written informed consent. The study was conducted in accordance with the ethical principles of the Declaration of Helsinki.

Blood samples and DNA isolation. The blood samples used in the study were collected from the Institute of Biomedical 


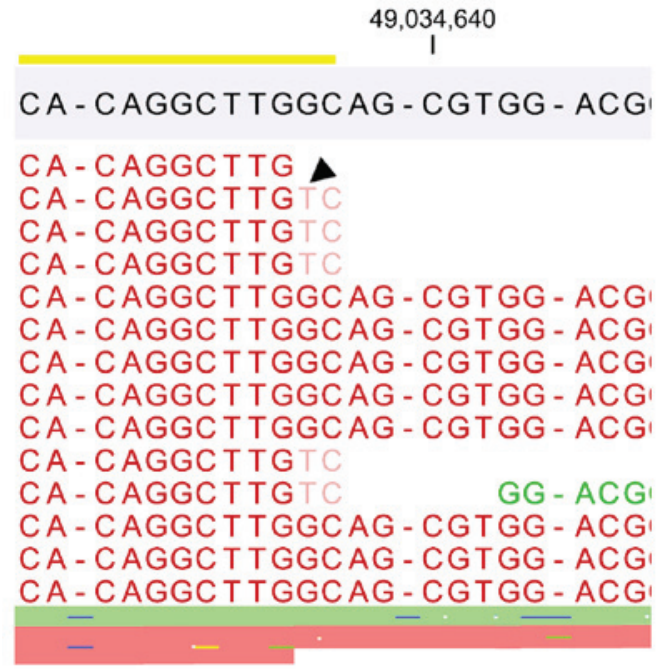

Figure 1. Possible primer-related sequencing error. The primer (shown in yellow underline) bound one of the non-specific sites, resulting in a sequencing error of $\mathrm{G}$ to $\mathrm{T}$ transversion mutation in PRICKLE3 (p. Cys251Ter, black arrowhead) located on chromosome $\mathrm{X}$. This error was confirmed by Sanger sequencing.

Research and Innovation Hospital (Kobe, Japan). DNA was isolated from peripheral blood mononuclear cells using the QIAamp DNA Blood Mini kit (Qiagen, Hilden, Germany), as per the manufacturer's instructions. We measured DNA concentration with NanoDrop Lite Spectrophotometer (Thermo Fisher Scientific, Inc., Waltham, MA, USA).

Ion Torrent Proton library preparation and sequencing. An Ion Torrent adapter-ligated library was generated following the manufacturer's protocol (Ion AmpliSeq ${ }^{\mathrm{TM}}$ Exome RDY kit PIv3, Rev. A.0; MAN0010084; Thermo Fisher Scientific, Inc.). Briefly, $100 \mathrm{ng}$ high-quality genomic DNA was used to prepare the Ion AmpliSeq ${ }^{\mathrm{TM}}$ Exome capture library. Pooled amplicons were end-repaired, and Ion Torrent adapters and amplicons were ligated with DNA ligase. Following AMPure bead purification (Beckman Coulter, Inc., Brea, CA, USA), the concentration and size of the library were determined using the Applied Biosystems ${ }^{\circledR}$ StepOne ${ }^{\mathrm{TM}}$ Real-Time PCR system and Ion Library TaqMan ${ }^{\circledR}$ Quantitation kit (both from Thermo Fisher Scientific, Inc.).

Sample emulsion PCR, emulsion breaking, and enrichment were performed using the Ion $\mathrm{PI}^{\mathrm{TM}} \mathrm{Hi}-\mathrm{Q}^{\mathrm{TM}}$ Chef 200 kit (Thermo Fisher Scientific, Inc.), according to the manufacturer's instructions. An input concentration of one DNA template copy per ion sphere particles (ISPs) was added to the emulsion PCR master mix and the emulsion was generated using the Ion Chef ${ }^{\text {TM }}$ System (Thermo Fisher Scientific, Inc.). Template-positive ISPs were enriched, sequencing was performed using Ion $\mathrm{PI}^{\mathrm{TM}}$ Chip kit v3 chips on the Ion Torrent Proton, and barcoding was performed using the Ion DNA Barcoding kit (Thermo Fisher Scientific, Inc.).

Variant calling. Data from the Proton runs were initially processed using Ion Torrent platform-specific pipeline software, Torrent Suite v4.0 (Thermo Fisher Scientific, Inc.) to generate sequence reads, trim adapter sequences, filter, and remove poor signal-profile reads. Initial variant calling from
A
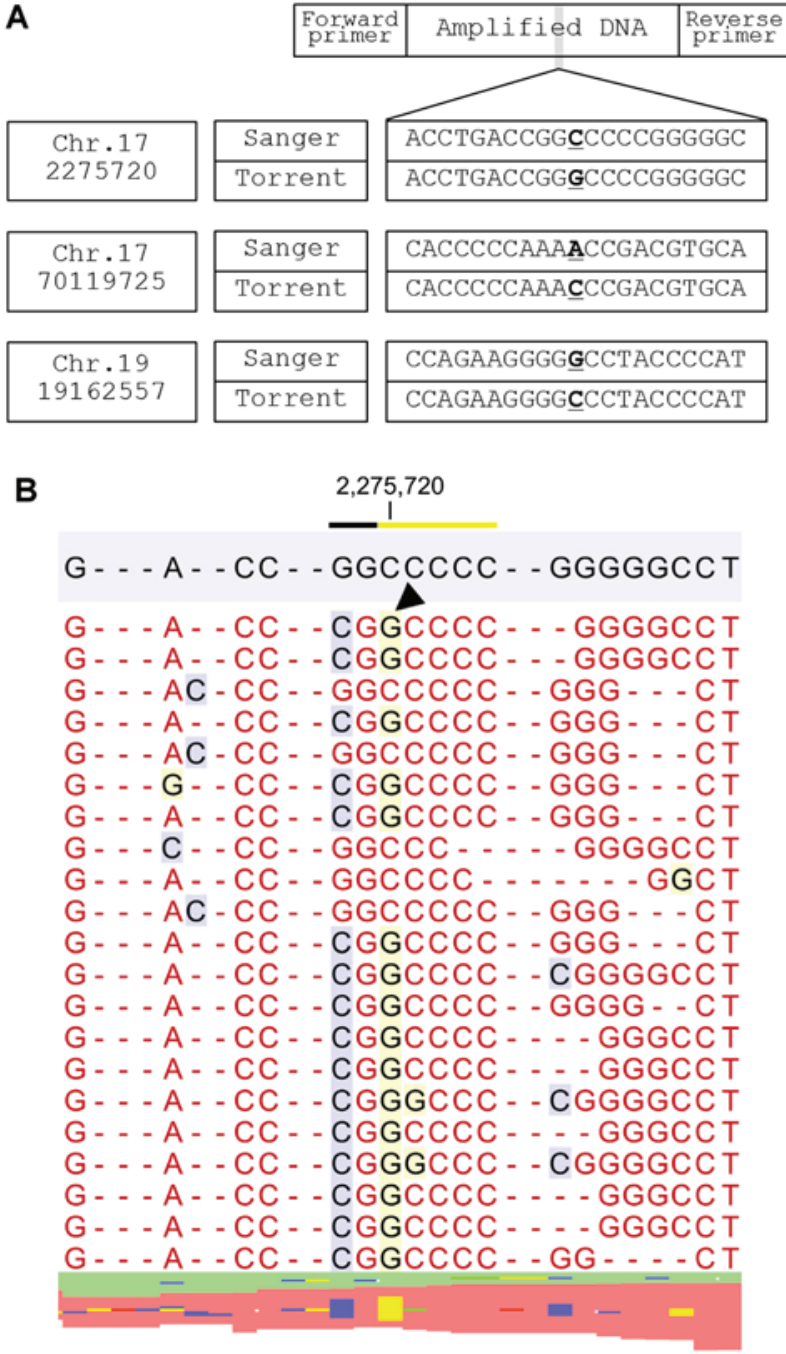

Figure 2. Sequence errors categorized into Group 1. (A) Position of the variants, results of Sanger sequencing and sequence error of the Ion Torrent system are shown. (B) Representative mapped lesion of a $\mathrm{C}>\mathrm{G}$ transversion in chromosome 17 position 2275720 (g.17:2275720 C>G hg19). Two homopolymer lesions (black and yellow underline) are directly linked in the hg19 reference sequence. A sequence error occurred in junctional area (black arrowhead).

the Ion AmpliSeq ${ }^{\mathrm{TM}}$ sequencing data was generated using Torrent Suite with a plug-in 'variant caller' program. To eliminate erroneous base calling, three filtering steps were used to generate final variant calling. The first filter was set at an average depth of total coverage of $>50$, an each variant coverage of $>15$, and $\mathrm{P}<0.01$. The second filter was employed by visually examining mutations using Integrative Genomics Viewer software (http://www.broadinstitute.org/igv) or CLC Genomics Workbench version 9.5.1 (Qiagen), as well as by filtering out possible strand-specific errors (i.e., a mutation was detected only in one, but not both, strands of DNA).

\section{Results}

A total of 26,260 SNVs were initially detected. We randomly selected 98 SNVs to be validated by Sanger sequencing. Of these, nine single nucleotide sequence errors were identified. A previous study identified primer-related sequencing errors 
A

\begin{tabular}{|c|c|c|c|}
\hline & $\begin{array}{l}\text { Forw } \\
\text { prin }\end{array}$ & Amplified DNA & $\begin{array}{l}\text { Revers } \\
\text { primer }\end{array}$ \\
\hline \multirow{2}{*}{$\begin{array}{c}\text { Chr. } 4 \\
76943088\end{array}$} & Sanger & \multicolumn{2}{|c|}{ TGGTTTTAAGGAGATCTTTTA } \\
\hline & Torrent & \multicolumn{2}{|c|}{ TGGTTTTAAGTAGATCTTTTA } \\
\hline \multirow{2}{*}{$\begin{array}{c}\text { Chr.6 } \\
15508582\end{array}$} & Sanger & \multicolumn{2}{|c|}{ AGTCACTATT $\underline{\mathbf{c} C C T G G C T A A A}$} \\
\hline & Torrent & \multicolumn{2}{|c|}{ AGTCACTATTACCTGGCTAAA } \\
\hline \multirow{2}{*}{$\begin{array}{c}\text { Chr.12 } \\
19522629\end{array}$} & Sanger & \multicolumn{2}{|c|}{ TGTTTTAGTGAAAAGTCTGTC } \\
\hline & Torrent & \multicolumn{2}{|c|}{ TGTTTTAGTGTAAAGTCTGTC } \\
\hline \multirow{2}{*}{$\begin{array}{c}\text { Chr.X } \\
49034636\end{array}$} & Sanger & \multicolumn{2}{|c|}{ CACAGGCTTGGCAGCGTGGAC } \\
\hline & Torrent & \multicolumn{2}{|c|}{ CACAGGCTTGTCAGCGTGGAC } \\
\hline
\end{tabular}

B

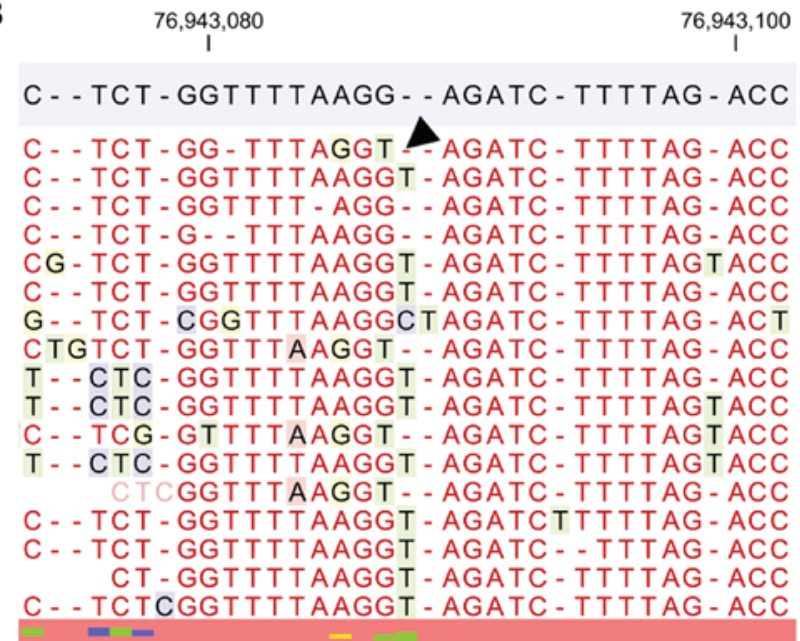

Figure 3. Sequence errors categorized into Group 2. (A) Position of the variants, results of Sanger sequencing and sequence error of the Ion Torrent system are shown. (B) Representative mapped lesion of a $\mathrm{G}>\mathrm{T}$ transversion in chromosome 4 position 76943088 (g.4:76943088 G>T hg19). One nucleotide loss of homopolymer area is occupied by another nucleotide (black arrowhead).

in the Ion Torrent system (9). Of the 18 total primers (forward and reverse) of the exome capture kit we analyzed, one possible primer-related error was observed (Fig. 1).

The rest of the sequencing errors were unrelated to primers and were classified into three groups. Group 1 sequencing errors result from two homopolymer lesions, on both the $5^{\prime}$ and $3^{\prime}$ sides, which cause a sequence error in the nucleotides of the junctional area (Fig. 2). NGS showed that loss of a nucleotide in one homopolymer area was accompanied by gain of a nucleotide in the other homopolymer area. The insertion locus was either at the immediate ends of the homopolymer regions (e.g., where sequence 'AAAAGGGG' is reported as 'AAAA $\underline{A} G G G$ ') or at a location a few nucleotides downstream of a homopolymer region (e.g., where 'AAAAACCGT' becomes 'AAAAACACGT' and the left-side ' $\mathrm{C}$ ' is masked, resulting in a sequencing error).

Group 2 sequencing errors occur when a nucleotide lost from a homopolymer area is replaced by a nucleotide from another homopolymer within 10 nucleotides of the observed

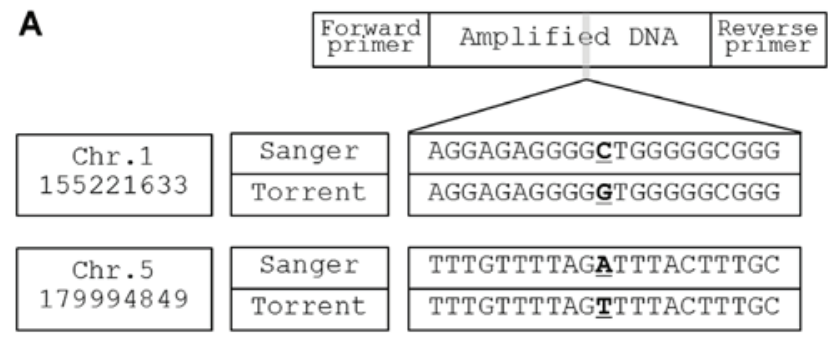

B

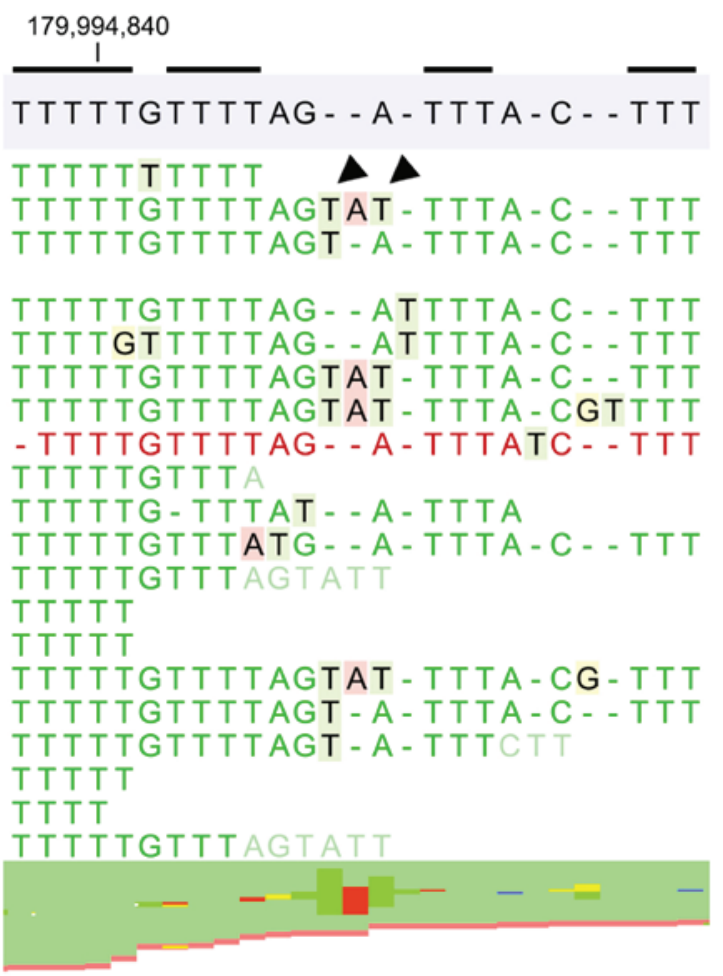

Figure 4. Sequence errors categorized into Group 3. (A) Position of the variants, results of Sanger sequencing and sequence error of the Ion Torrent system are shown. (B) Representative mapped lesion of an $\mathrm{A}>\mathrm{T}$ transversion in chromosome 5 position 179994849 (g.5:179994849 A>T hg19). Two lines of nucleotide (black arrowheads), which seem to originate from the neighborhood homopolymer area of thymidine (shown with black line), are inserted. These nucleotides mask true sequence and induce sequence errors as a result.

sequence error (Fig. 3). The neighboring homopolymer lesion is considered to be the main cause of this sequence error.

In Group 3, sequence error originates from the 'elongation' of a homopolymer lesion which may replace adjacent nucleotides (e.g., GGGCTA becomes GGGGTA). Insertion of two nucleotides, multiple homopolymers of which exist around the error, are observed in the sequence-error site. These two nucleotides mask the true nucleotide and a sequencing error occurs as a result (Fig. 4). Notably, all of the false-positive SNVs identified in the study are related to homopolymer regions.

\section{Discussion}

The Ion Torrent system uses semiconductor sequencing technology to detect the protons that are released as nucleotides incorporated during DNA synthesis (5). DNA fragments with specific adapter sequences are linked and then clonally amplified by emulsion PCR on the surface of $3-\mu \mathrm{m}$ beads. These 
templated beads are loaded into proton-sensing microwells fabricated on a silicon wafer, and sequencing is primed from a specified location in the adapter sequence. A microwell is then flooded with a single species of deoxyribonucleotide triphosphate (dNTP). If the introduced dNTP is complementary to the leading template nucleotide, it is incorporated into the growing complementary strand (10). This causes the release of a proton, which triggers an ion-sensitive transistor sensor. If homopolymer repeats are present in the template sequence, multiple dNTP molecules may be incorporated in a single cycle, which leads to a corresponding number of released hydrogens and a proportionally higher electronic signal. This signal intensity is theoretically proportional to the number of released nucleotides; however, miscount of the intensity can occur, causing what is termed a homopolymer error. The majority of homopolymer errors occur at the immediate ends of homopolymer regions, where a sequence such as 'AAAAAAAA' (an 8-nucleotide repeat) is reported as 'AAAAAAAAA' (a 9-nucleotide repeat) or 'AAAAAAA' (a 7-nucleotide repeat). These errors can also occur at a location several nucleotides downstream from a homopolymer region. In these cases, a sequence such as 'AAAAAATGC' is read as 'AAAAAATAGC'. Alternatively, a sequence of 'AAAAGTCG' may be recognized as 'AAAAATCG' or 'AAACGTCG'.

Sequencing errors remain a major challenge in the analysis of SNVs using NGS platforms. Our results indicated that SNVs located on junctional areas of two homopolymers (Group 1) require confirmation with Sanger sequencing. SNVs originating from homopolymer shortening or elongation (Groups 2 and 3, respectively) are possibly false positives, and confirmation is necessary. Several strategies are being investigated to avoid these homopolymer sequencing errors. The Hi-Q enzyme was introduced in 2014 to enhance SNV and indel performance on the Ion PGM system and later on the Ion Proton System. Internal tests indicated that the Ion PGM system used with the Hi-Q kit achieved a $43 \%$ reduction of indel errors in amplicon sequencing over the previous Ion PGM sequencing enzyme. Another strategy is the development of software for variant detection. Ion Reporter software is designed for analyzing sequence data obtained from the Ion Torrent system. This software is based on the algorithm of FreeBayes, a Bayesian genetic variant detector designed to find small polymorphisms (11).

The present study revealed that the majority of the SNV sequencing errors originate from homopolymer insertion/deletion errors, are far more common in the Ion Torrent system than the competitor Illumina system. Even when the abovementioned measures are employed, the results obtained using the Ion Reporter System should be interpreted with caution.

\section{Acknowledgements}

The present study was supported by JSPS KAKENHI grant no. JP15K10017.

\section{References}

1. Pleasance ED, Cheetham RK, Stephens PJ, McBride DJ, Humphray SJ, Greenman CD, Varela I, Lin ML, Ordóñez GR, Bignell GR, et al: A comprehensive catalogue of somatic mutations from a human cancer genome. Nature 463: 191-196, 2010.

2. Horak P, Frohling $\mathrm{S}$ and Glimm H: Integrating next-generation sequencing into clinical oncology: strategies, promises and pitfalls. ESMO Open 1: e000094, 2016.

3. Ng SB, Buckingham KJ, Lee C, Bigham AW, Tabor HK, Dent KM, Huff CD, Shannon PT, Jabs EW, Nickerson DA, et al: Exome sequencing identifies the cause of a mendelian disorder. Nat Genet 42: 30-35, 2010.

4. Bonnefond A, Durand E, Sand O, De Graeve F, Gallina S, Busiah K, Lobbens S, Simon A, Bellanné-Chantelot C, Létourneau L, et al: Molecular diagnosis of neonatal diabetes mellitus using next-generation sequencing of the whole exome. PLoS One 5: e13630, 2010.

5. Rothberg JM, Hinz W, Rearick TM, Schultz J, Mileski W, Davey M, Leamon JH, Johnson K, Milgrew MJ, Edwards M, et al: An integrated semiconductor device enabling non-optical genome sequencing. Nature 475: 348-352, 2011.

6. Boland JF, Chung CC, Roberson D, Mitchell J, Zhang X, Im KM, He J, Chanock SJ, Yeager $M$ and Dean M: The new sequencer on the block: Comparison of Life Technology's Proton sequencer to an Illumina HiSeq for whole-exome sequencing. Human genetics 132: 1153-1163, 2013.

7. Zhang G, Wang J, Yang J, Li W, Deng Y, Li J, Huang J, Hu S and Zhang B: Comparison and evaluation of two exome capture kits and sequencing platforms for variant calling. BMC genomics 16: $581,2015$.

8. Damiati E, Borsani G and Giacopuzzi E: Amplicon-based semiconductor sequencing of human exomes: performance evaluation and optimization strategies. Human genetics 135: 499-511, 2016.

9. McCall CM, Mosier S, Thiess M, Debeljak M, Pallavajjala A, Beierl K, Deak KL, Datto MB, Gocke CD, Lin MT and Eshleman JR: False positives in multiplex PCR-based nextgeneration sequencing have unique signatures. JMD 16: 541-549, 2014.

10. Thermo Fisher Scientific, Ion Torrent. https://www.thermofisher. com/jp/en/home/brands/ion-torrent.html. Accessed April 10, 2017.

11. Garrison E and Marth G: Haplotype-based variant detection from short-read sequencing. arXiv.org>q-bio>arXiv:1207.3907, 2012 . 